\title{
Diagnostic efficacy of FibroScan for liver inflammation in patients with chronic hepatitis B: a single-center study with 1185 liver biopsies as controls
}

\author{
Kaiping Jiang ${ }^{1 *+}$, Lei Zhang ${ }^{1 \dagger}$, Jianhong $\mathrm{Li}^{1 \dagger}{ }^{\dagger}$, Hongtao Hu${ }^{1}$, Qinghua Huang ${ }^{1}$, Tengyu Qiu ${ }^{1}$, Xiaoai Mo ${ }^{1}$, \\ Jian Ren ${ }^{1}$, Wenqiang Guo ${ }^{1}$, Yin Tao ${ }^{1}$, Haijun Cui ${ }^{1}$, Ying Zuo ${ }^{1}$, Xuli Chen ${ }^{1}$, Youqing Xie ${ }^{1}$, Yanxing Li ${ }^{1}$, \\ Haimin Liang ${ }^{1}$, Zhaohong Liu², Le Xie ${ }^{3}$, Rongjun Mao ${ }^{3}$, Qunfang Jiang ${ }^{1}$ and Kaizhou Huang ${ }^{1}$
}

\begin{abstract}
Background: Noninvasive diagnostic technologies that can dynamically monitor changes in liver inflammation are highly important for the management of chronic hepatitis B (CHB) patients and thus warrant further exploration. This study assessed the diagnostic efficacy of FibroScan for liver inflammation in CHB patients.

Methods: A total of 1185 patients were selected, and ultrasound-guided liver biopsy was performed within 1 month after the FibroScan test. The liver stiffness measurement (LSM), the reliability criteria (IQR/M) of LSM, the quality of liver biopsy (complete portal area, PA), and the liver inflammation grades were the main observation items of this study. With liver biopsy as the control, the diagnostic efficacy of FibroScan for liver inflammation in CHB patients was evaluated by receiver operating characteristic (ROC) curve analysis.

Results: The grade of liver inflammation was positively correlated with the stage of fibrosis $(r h o=0.829, P<0.001)$. Different grades of inflammation will have significant rise in LSM values within the same fibrosis stage, and LSM values were positively correlated with liver inflammation grade and fibrosis stage, and the rho is 0.579 and 0.593 respectively $(P<0.001)$. Significant differences in the LSM of FibroScan were observed among different grades of liver inflammation $(P<0.0001)$. Liver biopsy $(P A>10)$ served as the control, and the cutoff point and the area under ROC curves (AUCs) of the LSMs for different inflammation grades were as follows: G2, 8.6 kPa, 0.775; G3 9.8 kPa, 0.818; and G4, $11.0 \mathrm{kPa} ; 0.832$. With LSM cutoff values of $8.6 \mathrm{kPa}, 9.8 \mathrm{kPa}$ and $11.0 \mathrm{kPa}$, FibroScan showed certain diagnostic value for CHB patients with $G 2, G 3$ and $G 4$ liver inflammation, especially those with G4 inflammation.

Conclusions: The grade of liver inflammation was positively correlated with the stage of fibrosis, different grades of inflammation will have significant rise in LSM values within the same fibrosis stage. In addition to liver fibrosis, FibroScan could evaluate liver inflammation in CHB patients in a noninvasive manner.
\end{abstract}

\footnotetext{
*Correspondence: jkpingfs@vip.163.com

${ }^{\dagger}$ Co-first authors who contributed equally to this work: Kaiping Jiang, Lei

Zhang, Jianhong Li

${ }^{1}$ Department of Hepatology, Foshan Hospital of Traditional Chinese

Medicine Affiliated to Guangzhou University of Chinese Medicine, No.6

Qinren Road, Chancheng District, Foshan 528000, Guangdong Province,

China

Full list of author information is available at the end of the article
}

(C) The Author(s) 2022. Open Access This article is licensed under a Creative Commons Attribution 4.0 International License, which permits use, sharing, adaptation, distribution and reproduction in any medium or format, as long as you give appropriate credit to the original author(s) and the source, provide a link to the Creative Commons licence, and indicate if changes were made. The images or other third party material in this article are included in the article's Creative Commons licence, unless indicated otherwise in a credit line to the material. If material is not included in the article's Creative Commons licence and your intended use is not permitted by statutory regulation or exceeds the permitted use, you will need to obtain permission directly from the copyright holder. To view a copy of this licence, visit http://creativecommons.org/licenses/by/4.0/. The Creative Commons Public Domain Dedication waiver (http://creativeco mmons.org/publicdomain/zero/1.0/) applies to the data made available in this article, unless otherwise stated in a credit line to the data. 
Keywords: Noninvasive diagnosis, Chronic hepatitis B (CHB), Liver inflammation, FibroScan, Liver stiffness measurement (LSM)

\section{Background}

Chronic hepatitis B virus (HBV) infection, which causes nearly one million deaths each year, remains a major public health problem worldwide $[1,2]$. The 69th World Health Assembly approved a Global Health Sector Strategy to eliminate viral hepatitis by 2030 after the World Health Organization (WHO) issued its first ever guidelines for the prevention, care and treatment of persons with chronic hepatitis B (CHB) infection. A modeling study estimated that the global prevalence of HBsAg was $3.9 \%$ in 2016 [2]. Among untreated patients with $\mathrm{CHB}$ virus infection, 15-40\% progress to cirrhosis, which may lead to liver failure and liver cancer [3]. The prevention and treatment of CHB is so urgent that, in addition to drug research, researchers must explore rapid, dynamic and noninvasive diagnostic methods that could be used to monitor the occurrence and development of CHB. Noninvasive analyses of liver fibrosis might offer a promising strategy for earlier diagnosis [4], so noninvasive methods to evaluate liver fibrosis have been attempted. The most commonly used is transient elastography (TE), which estimates liver fibrosis by measuring liver stiffness [5]. Currently, FibroScan, which is based on TE techniques, is widely used across the globe and has become an important method for the assessment of liver fibrosis in patients with CHB [6-8]. The vast majority of patients with $\mathrm{CHB}$ will develop HBV-induced necrotic inflammation and progressive fibrotic liver processes [7], and patients with immune-active $\mathrm{CHB}$ display elevated alanine aminotransferase (ALT) activity and active hepatic necroinflammation [9], so the results of TE may be confounded by the severe inflammation associated with high ALT levels $[10,11]$. The LSM value obtained with FibroScan was also found to correlate significantly with both liver fibrosis and necroinflammatory activity on biopsy, which was considered to explain the TE measurement of TE [12].

Some authors have stated that TE cutoffs should incorporate ALT levels, which fluctuate with inflammation in HBV infection [13]. In this case, why not evaluate the potential of FibroScan for the diagnosis of liver inflammation in CHB patients? Thus, based on the data of 1185 liver biopsy specimens, we conducted a single-center large sample study to assess the value of FibroScan for the diagnosis of liver inflammation in patients with $\mathrm{CHB}$.

\section{Methods \\ Study design and patients}

The study protocol was approved by the Ethics Committee of Foshan Hospital of Traditional Chinese Medicine ([2016]006). All patients with CHB signed informed consent. All the data related to this study were registered on the International Clinical Trial Registry Platform (ChiCTR- DRD-16009773).

The study was carried out at Foshan Hospital of Traditional Chinese Medicine, Guangzhou University of Chinese Medicine, China (from May 2011 to May 2016). A total of 1185 patients with $\mathrm{CHB}$ were selected from the Department of Hepatology according to the clinical practice guidelines $[7,14]$. Patients with any of the following were excluded: liver cirrhosis or liver cancer; high levels of total bilirubin (TBIL) $(>150 \mu \mathrm{mol} /)$ or liver failure; complicated by metabolic diseases or autoimmune liver diseases; coinfected with HIV, HCV and HDV; abused alcohol or illegal drugs; a history of using nucleoside analogs, interferon, or other anti-hepatic fibrosis drugs within 24 weeks; receiving treatment with anti-inflammatory agents, hepatoprotectants or related drugs; mental diseases or other serious viscera diseases; overweight or central obesity patients $\left(\mathrm{BMI} \geq 28.0 \mathrm{~kg} / \mathrm{m}^{2}\right)$; pregnant or lactating women.

ALT (normal range: 0-40 U/L) and TBIL (normal range: $0-17 \mu \mathrm{mol} / \mathrm{L}$ ) were tested with an automatic biochemical analyzer, hepatitis B surface antigen (HBsAg) and hepatitis $\mathrm{B}$ e antigen ( $\mathrm{HBeAg}$ ) were detected by electrochemiluminescence immunoassays, and HBV DNA was analyzed via real-time PCR (detection limit: $2 \log _{10}$ $\mathrm{IU} / \mathrm{mL})$.

\section{FibroScan}

FibroScan ${ }^{\circledR} 502$ (Echosens, Paris, France) test was performed on an empty stomach in the morning or more than $2 \mathrm{~h}$ after food intake in patients with CHB. FibroScan was performed independently by the 3 operators with medical background in our department. They had been trained by Echosens and obtained the training certificate.Each operator had more than 500 times of successful operation experience. The median value of 10 effective measurements was successfully tested 10 times [15]. The LSM results are expressed in kilopascals $(\mathrm{kPa})$. In this study, the operators adhered to the following reliability criteria [16]: ratio of the interquartile range (IQR) to the median $(\mathrm{M})(\mathrm{IQR} / \mathrm{M})$ was less than 0.30 , with less 
than 0.10 being regarded as the best, and a success rate no less than $60 \%$, with over $90 \%$ being regarded as the best.

\section{Liver biopsy}

Ultrasound-guided liver biopsy was performed within 1 month after the FibroScan test had been completed. A 16-gauge disposable needle was used for the liver biopsy so that the length of the extracted liver tissue was greater than $1.5 \mathrm{~cm}$ and included at least 6 complete portal areas (PAs). The obtained liver tissue samples were fixed with $10 \%$ neutral formaldehyde solution, embedded in paraffin, and sliced into 5 pieces continuously. Routine HE staining, Masson staining and reticular fiber staining were used for diagnosis. The liver fibrosis stage was determined according to the METAVIR system ( $\mathrm{S}=$ fibrosis) [17]: $\mathrm{S} 0=$ no fibrosis, $\mathrm{S} 1=$ portal fibrosis without septa, $\mathrm{S} 2=$ portal fibrosis with rare septa, S3=numerous septa without cirrhosis, and $\mathrm{S} 4=$ cirrhosis.

According to the Scheuer scoring system [18], liver inflammation in the patients with $\mathrm{CHB}$ was classified into five grades: G0, G1, G2, G3 and G4. Moreover, the degree of hepatic steatosis was divided into four grades [19]: 0 (<5\%), 1 (mild, 5-33\%), 2 (moderate, 34-66\%), and 3 (severe, $>66 \%$ ).

The pathological diagnosis of all liver biopsy samples was completed by 2 pathologists in our hospital. If the independent pathological diagnosis results given by the two pathologists were consistent, a pathological report would be provided. If the results of the two pathologists were inconsistent, the director pathologist would give the final pathological diagnosis and provide a report after the two pathologists reviewed the pathological section of liver tissue and discussed it together.

\section{Statistical analysis}

Statistical analysis was carried out by SPSS 20.0. Categorical variables are presented as absolute (n) and relative (\%) frequencies, and continuous variables are presented as the means $\pm \mathrm{SD}$. The significance of each baseline difference was determined by the chi-square test, Fisher's exact test, unpaired t-test, or Mann-Whitney's test, as appropriate. A two-sided $P$ value of less than 0.05 was considered to indicate statistical significance. The correlations were analyzed with Pearson's correlation and the test of Spearman's rank-correlation coefficient.

Based on the gold standard for the pathological grade of liver biopsy tissue and carried out by MedCalc, the receiver operating characteristic (ROC) curve was plotted, and the area under ROC curve (AUC), cutoff-off point, sensitivity, specificity and false positive rate were calculated, respectively, to determine the efficiency of the LSM by FibroScan in diagnosing the degree of liver inflammation. The data were artificially divided into two parts. We considered $G=1$ to be relatively healthy and $\mathrm{G}=2,3$, and 4 to be diseased. The AUCs were all between 1.0 and 0.5. An AUC between 0.5 and 0.7 was regarded as low accuracy, an AUC between 0.7 and 0.9 was regarded as moderate accuracy, and an AUC was above 0.9 was regarded as high accuracy; an AUC equal to 0.5 indicated no diagnostic value.

\section{Results}

\section{Patients}

The main demographic and clinical characteristics of the 1185 patients with CHB included in the study are presented in Table 1. Among them, there were 894 (75\%) male patients, 291 (25\%) female patients, 658 cases of $\mathrm{HBeAg}$-positive $\mathrm{CHB}$ and 527 cases of HBeAg-negative $\mathrm{CHB}$. The median age of the HBeAg-negative group was 37 years, which was older than that of the HBeAg-positive group (31 years) $(P<0.001)$. The majority of patients were $\mathrm{HBeAg}$ positive or negative $(P=0.012)$. Among the 273 patients with hepatic steatosis confirmed by liver biopsy, not only was the incidence of hepatic steatosis in men $(\mathrm{n}=230,84 \%)$ higher than that in women $(\mathrm{n}=43$, 16\%) $(P<0.001)$ but also the incidence of hepatic steatosis in the HBeAg-negative group $(\mathrm{n}=142,27 \%)$ was higher than that in the HBeAg-positive group $(n=131,20 \%)$ $(P=0.004)$.

The mean \pm SD of ALT, which was higher $(169 \pm 216$ $\mathrm{U} / \mathrm{L}$ ) in the HBeAg-positive patients than in the HBeAgnegative patients $(133 \pm 220 \mathrm{U} / \mathrm{L}) \quad(P=0.005)$, was $153 \pm 218 \mathrm{U} / \mathrm{L}$ in all patients. The levels of TBIL in 1010 patients (85\%) were lower than $17 \mu \mathrm{mol} / \mathrm{L}$, those in 166 patients $(14 \%)$ were between 17 and $50 \mu \mathrm{mol} / \mathrm{L}$, those in 6 patients $(0.5 \%)$ were between 51 and $100 \mu \mathrm{mol} / \mathrm{L}$, and those in 3 patients $(0.25 \%)$ were between 101 and $150 \mu \mathrm{mol} / \mathrm{L}$. The level of HBV DNA (mean \pm SD) in the $\mathrm{HBeAg}$-positive patients was higher $\left(5.97 \pm 1.96 \log _{10} \mathrm{IU} /\right.$ $\mathrm{mL})$ than that $\left(4.97 \pm 1.43 \log _{10} \mathrm{IU} / \mathrm{mL}\right)$ in the $\mathrm{HBeAg}$ negative patients $(P<0.001)$.

\section{FibroScan}

Based on a FibroScan test success rate of over $90 \%$, the LSM values ranged from 2.4 to $72 \mathrm{kPa}$, with an average value of $11.96 \mathrm{kPa}$, and the LSM reliability results of IQR/M (\%) were $70 \%(\leq 0.10), 23 \%(0.10-0.15), 4 \%$ $(0.15-0.20)$, and $3 \%(0.20-0.30)$.

\section{Liver biopsy}

Among the liver biopsy tissues of 1185 patients with CHB, 977 cases (82\%) had more than 10 PAs, and 208 cases $(18 \%)$ had fewer than 10 PAs; there was no statistically significant difference in terms of $\operatorname{sex}(P=0.152)$. The inflammation grade and fibrosis stage of the liver tissues 
Table 1 Demographic and clinical characteristics of the CHB patients

\begin{tabular}{|c|c|c|c|c|c|}
\hline & \multirow[b]{2}{*}{ Total $(n=1185)$} & \multicolumn{2}{|l|}{ HBeAg } & \multicolumn{2}{|c|}{ hepatic steatosis } \\
\hline & & Positive $(n=658)$ & Negative $(n=527)$ & $n=273$ & $P$ \\
\hline \multicolumn{6}{|l|}{ Age } \\
\hline Median years & $33(15-67)$ & $31(15-60)$ & $37(17-67)$ & $36(16-67)$ & \\
\hline Means $\pm S D$ & $33 \pm 9$ & $31 \pm 8^{A}$ & $37 \pm 9^{A}$ & $36 \pm 9$ & $<.001^{\mathrm{A}}$ \\
\hline \multicolumn{6}{|l|}{ Sex } \\
\hline Female & $291(25 \%)$ & $180(27 \%)^{B}$ & $111(21 \%)^{B}$ & $43(16 \%)^{C}$ & $.012^{\mathrm{B}}$ \\
\hline Male & $894(75 \%)$ & $478(73 \%)^{B}$ & $416(79 \%)^{B}$ & $230(84 \%)^{C}$ & $<.001^{\mathrm{C}}$ \\
\hline \multicolumn{6}{|l|}{$A L T(U / L)$} \\
\hline Means $\pm S D$ & $153 \pm 218$ & $169 \pm 216^{D}$ & $133 \pm 220^{D}$ & & $.005^{\mathrm{D}}$ \\
\hline \multicolumn{6}{|l|}{ TBIL $(\mu \mathrm{mol} / \mathrm{L})$} \\
\hline$<17$ Hmol/L (n) & $1010(85 \%)$ & & & & \\
\hline 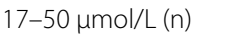 & $166(14 \%)$ & & & & \\
\hline $51-100 \mu \mathrm{mol} / \mathrm{L}(\mathrm{n})$ & $6(0.5 \%)$ & & & & \\
\hline $101-150 \mu \mathrm{mol} / \mathrm{L}(\mathrm{n})$ & $3(0.25 \%)$ & & & & \\
\hline \multicolumn{6}{|l|}{ HBV DNA $\left(\log _{10} I U / L\right)$} \\
\hline Means $\pm S D$ & $5.97 \pm 1.96$ & $6.77 \pm 1.14^{\mathrm{E}}$ & $4.97 \pm 1.43^{\mathrm{E}}$ & & $<.001^{\mathrm{E}}$ \\
\hline Hepatic steatosis & $273(23 \%)$ & $131(20 \%)^{F}$ & $142(27 \%)^{F}$ & & $.004^{\mathrm{E}}$ \\
\hline \multicolumn{6}{|c|}{ 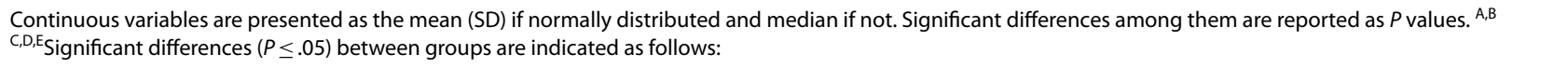 } \\
\hline \multicolumn{6}{|c|}{${ }^{A}$ For comparison between $\mathrm{HBeAg}$-positive and $\mathrm{HBeAg}$-negative patients } \\
\hline \multicolumn{6}{|c|}{${ }^{B}$ For comparison of females and males who were $\mathrm{HBeAg}$ positive and $\mathrm{HBeAg}$ negative } \\
\hline \multicolumn{6}{|c|}{${ }^{\mathrm{C}}$ For comparison of $\mathrm{CHB}$ with hepatic steatosis between females and males } \\
\hline \multicolumn{6}{|c|}{${ }^{\mathrm{D}}$ For comparison of ALT between HBeAg-positive and HBeAg-negative patients } \\
\hline${ }^{\mathrm{F}}$ For comparison of $\mathrm{CH}$ & atic steatosis betwee & positive and $\mathrm{HBeAg}$ & & & \\
\hline
\end{tabular}

Table 2 Inflammation grade and fibrosis stage in liver tissue of CHB patients

\begin{tabular}{|c|c|c|c|c|}
\hline \multirow[t]{2}{*}{$S, n=1185$} & \multicolumn{4}{|c|}{$\mathrm{G}, \mathrm{n}=1185$} \\
\hline & G1 & G2 & G3 & G4 \\
\hline so & 42 & 13 & 1 & 0 \\
\hline S1 & 64 & 164 & 15 & 0 \\
\hline S2 & 10 & 220 & 148 & 0 \\
\hline S3 & 0 & 13 & 268 & 43 \\
\hline S4 & 0 & 1 & 43 & 140 \\
\hline
\end{tabular}

Spearman's rho $=0.829, P<0.001$ are shown in Table 2. From Table 2, we can see that the fibrosis stage and the inflammation grade are two-way ordered data, and the grade of liver inflammation was positively correlated with the stage of fibrosis (the Spearman's $r h o=0.829, P<0.001)$. Especially the liver inflammation of patients with cirrhosis (S4) is mostly G4, and G1 and G2 are rare. There was a statistically significant difference in the liver inflammation grade between the $\mathrm{PA} \geq 10$ group and $\mathrm{PA}<10$ group $(P<0.001)$ (Table 3$)$.

Hepatic steatosis was found in 1185 patients with $\mathrm{CHB}$ in 273 cases $(23 \%)$, of which 205 cases $(75 \%)$ were mild,

Table 3 The liver inflammation grade among CHB patients with different PAs

\begin{tabular}{|c|c|c|c|c|c|c|}
\hline \multirow[t]{2}{*}{ Group } & \multirow[t]{2}{*}{$\mathrm{n}$} & \multicolumn{4}{|c|}{$\mathbf{G}, \mathrm{n}$} & \multirow[t]{2}{*}{ Mean rank } \\
\hline & & 1 & 2 & 3 & 4 & \\
\hline$\geq 10 \mathrm{PA}$ & 977 & 60 & 321 & 421 & 175 & 634.31 \\
\hline$<10 \mathrm{PA}$ & 208 & 56 & 89 & 54 & 9 & 398.98 \\
\hline
\end{tabular}

Mann-Whitney $\cup Z=-9.548, P=.000$

There was a statistically significant difference between the $P A \geq 10$ group and $P A<10$ group in terms of liver inflammation grade $(P<0.001)$ 
50 cases were moderate (18\%), and 18 cases were severe (7\%). There was no significant difference in the inflammation grade $(P=0.082)$ or fibrosis stage $(P=0.177)$ between the $\mathrm{CHB}$ patients with hepatic steatosis and those without hepatic steatosis.

\section{ALT}

With $40 \mathrm{U} / \mathrm{L}$ as the baseline, the effect of ALT levels below $40 \mathrm{U} / \mathrm{L}$ and 2, 3, 5 and 10 times higher than the baseline on the LSM was observed. Differences in ALT levels did not affect the accuracy of LSM in the diagnosis of liver inflammation $(P>0.05)$.

\section{Changes of LSM values in different grades of inflammation within the same fibrosis stage}

In CHB patients with liver fibrosis at stage $\mathrm{S} 2$ or above, the LSM values rised with the increase of the grades of liver inflammation. In S2 stage of liver fibrosis, there was significant difference in LSM values between different grades of liver inflammation $(\mathrm{F}=10.664, P<0.001)$. There was significant difference in LSM values between G1 inflammation and G3 inflammation $(P=0.01)$ and that between G2 inflammation and G3 inflammation $(P<0.001)$. In the S3 stage of liver fibrosis, there was significant difference in LSM values between different grades of liver inflammation $(\mathrm{F}=6.194, P=0.002)$. There was no statistical difference in LSM values between G2 inflammation and G3 inflammation $(P=0.051)$, there was significant difference in LSM values between G2 inflammation and G4 inflammation $(P=0.002)$ and that between G3 inflammation and G4 inflammation $(P=0.006)$. There was significant difference in LSM values between G3 inflammation and G4 inflammation in S4 stage of liver fibrosis $(P<0.001)$ (Table 4). Further analysis of the correlation between liver fibrosis stage, liver inflammation grade and LSM values in patients with chronic hepatitis B showed that LSM values were positively correlated with liver inflammation grade and fibrosis stage, the Spearman's Rho was 0.579 and 0.593 , respectively $(P<0.001)$.

\section{The diagnostic efficacy of FibroScan (LSM) for liver inflammation in $1185 \mathrm{CHB}$ patients}

No G0 liver inflammation was observed in these $\mathrm{CHB}$ patients. The diagnostic efficacy of FibroScan (LSM, kPa) for liver inflammation was analyzed based on the sensitivity, specificity, false positive rate, cutoff points and AUCs for different inflammation grades (G1, G2, G3, G4).

- G1-G2G3G4 There were significant differences in the LSMs between the liver inflammation grades when stratified by G1-G2G3G4 $(P<0.0001)$. The sensitivity was 56.82 , the specificity was 83.62 , the false positive rate was 16.38, the LSM cutoff value for group G2 was 9.6, and the AUC was 0.743.

- G1G2-G3G4 There were significant differences in the LSMs between the liver inflammation grades when stratified by G1G2-G3G4 $(P<0.0001)$. The sensitivity was 74.36 , the specificity was 74.71 , the false positive rate was 20.69, the LSM cutoff value for group G3 was 9.7, and the AUC was 0.807.

- G1G2G3-G4 There were significant differences in the LSMs $(\mathrm{kPa})$ between the liver inflammation grades when stratified by G1G2G3-G4 $(P<0.0001)$. The sensitivity was 84.78 , the specificity was 70.33 , the false positive rate was 29.67 , the LSM cutoff value for group G4 was 11.4, and the AUC was 0.838 .

Table 4 Changes of LSM values in different grades of inflammation within the same fibrosis stage

\begin{tabular}{llrrr}
\hline $\mathbf{S}, \mathbf{n}=\mathbf{1 1 8 5}$ & $\mathbf{G} \mathbf{n}=\mathbf{1 1 8 5}$ & & \\
\cline { 2 - 5 } & G1 (LSM) & G2 (LSM) & G3 (LSM) & G4 (LSM) \\
\hline S0 (56) & $42(7.31 \pm 2.37)$ & $13(8.05 \pm 2.28)$ & $1(5.6)$ & 0 \\
S1 (243) & $64(7.99 \pm 2.54)$ & $164(8.22 \pm 3.34)$ & $15(8.49 \pm 2.77)$ & 0 \\
S2 (378) & $10(8.67 \pm 1.44)$ & $220(8.85 \pm 3.20)$ & $148(10.54 \pm 3.97)$ & 0 \\
S3 (325) & 0 & $13(9.89 \pm 2.66)$ & $268(13.97 \pm 6.87)$ & $44(17.24 \pm 10.24)$ \\
S4 (183) & 0 & $1(9.5)$ & $43(14.78 \pm 6.39)$ & $139(20.87 \pm 11.84)$ \\
\hline
\end{tabular}

S0 stage of fibrosis: $\mathrm{t}=-1.002, P=0.321$

S1 stage of fibrosis: $F=0.211, P=0.81$

S2 stage of fibrosis: $F=10.664, P<0.001 ; G 1$ versus $G 3, P=0.01 ; G 3$ versus $G 2, P<0.001$

S3 stage of fibrosis: $\mathrm{F}=6.194, P=0.002 ; \mathrm{G} 2$ versus $\mathrm{G} 3, P=0.051 ; \mathrm{G} 2$ versus $\mathrm{G} 4, P=0.002 ; \mathrm{G} 3$ versus $\mathrm{G} 4, P=0.006$

$\mathrm{S} 4$ stage of fibrosis: $\mathrm{G} 3$ versus $\mathrm{G} 4, \mathrm{t}=-4.358, P<0.001$ 
Table 5 The diagnostic efficacy of FibroScan (LSM) for liver inflammation or fibrosis when PA $\geq 10$

\begin{tabular}{|c|c|c|c|c|c|c|c|c|c|}
\hline LSM & Group & Cutoff (positive) & Sensitivity (\%) & Specificity (\%) & Youden index & AUC & SE & z & $P(A \cup C=0.5)$ \\
\hline \multirow[t]{7}{*}{$\mathrm{kPa}((\mathrm{PA} \geq 10)))$} & $\mathrm{G}=4 \mathrm{G}=1,2,3$ & $>11$ & 87.43 & 66.21 & 0.536 & 0.832 & 0.016 & 20.809 & $<0.0001$ \\
\hline & $\mathrm{G}=1,2 \mathrm{G}=3,4$ & $\leq 9.8$ & 75.59 & 75.17 & 0.508 & 0.818 & 0.013 & 23.905 & $<0.0001$ \\
\hline & $\mathrm{G}=1 \mathrm{G}=2,3,4$ & $\leq 8.6$ & 78.33 & 68.70 & 0.470 & 0.775 & 0.024 & 11.542 & $<0.0001$ \\
\hline & $S=0,1,2 S=3,4$ & $\leq 10.4$ & 77.45 & 73.88 & 0.513 & 0.826 & 0.013 & 24.667 & $<0.0001$ \\
\hline & $S=0 S=1,2,3,4$ & $\leq 8.6$ & 83.33 & 67.69 & 0.510 & 0.791 & 0.029 & 10.126 & $<0.0001$ \\
\hline & $S=4 S=0,1,2,3$ & $>11$ & 83.05 & 65.37 & 0.484 & 0.807 & 0.017 & 17.740 & $<0.0001$ \\
\hline & $\mathrm{S}=0,1 \mathrm{~S}=2,3,4$ & $\leq 8.8$ & 74.19 & 71.43 & 0.456 & 0.789 & 0.016 & 17.602 & $<0.0001$ \\
\hline
\end{tabular}

PA of liver tissue $\geq 10$ : There were significant differences in the LSMs among the liver inflammation or fibrosis grades $(P<0.0001)$
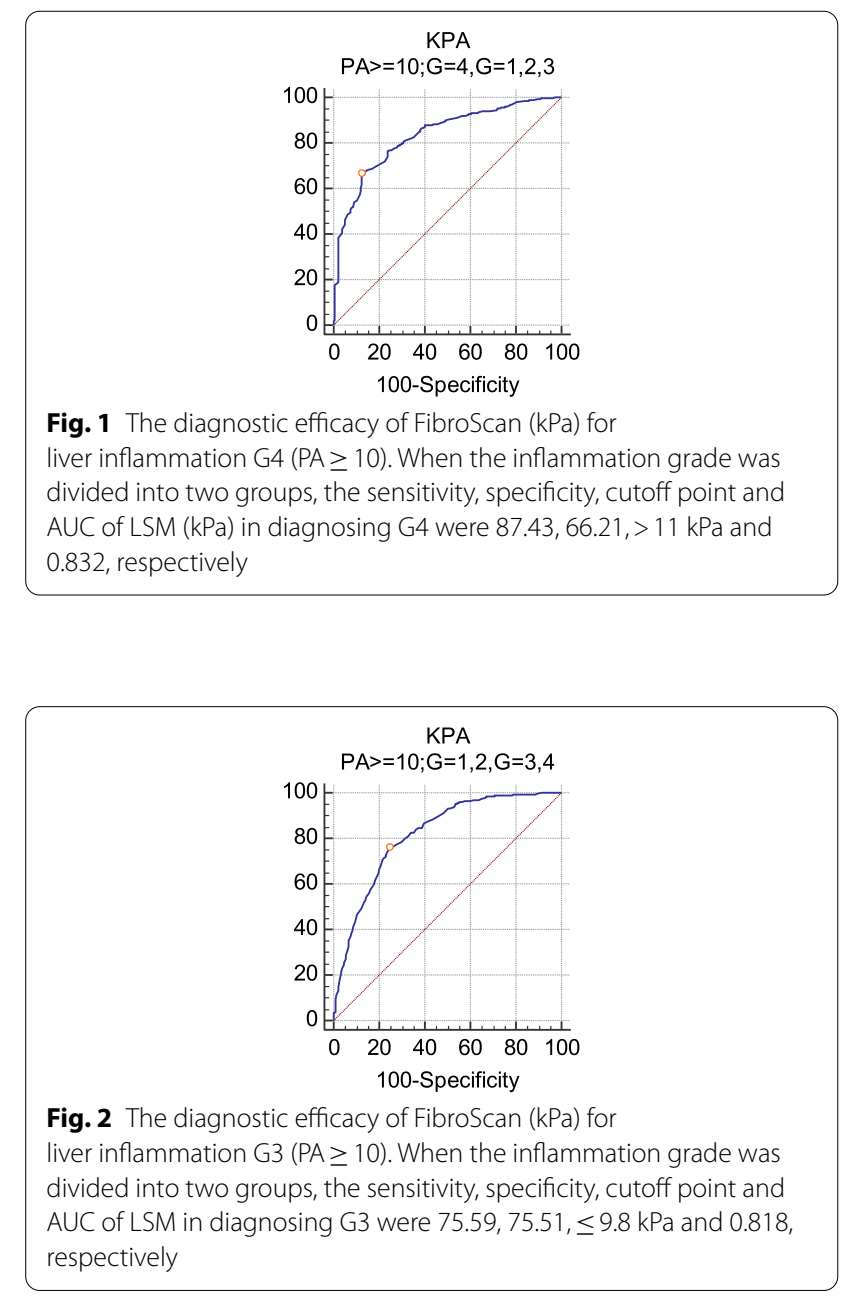

\section{The diagnostic efficacy of FibroScan ( $\mathrm{kPa}$ ) for liver inflammation based on different PAs in the liver tissue of $\mathrm{CHB}$ patients}

$\mathrm{PA} \geq 10$ : There were significant differences in the LSMs among the liver inflammation grades $(P<0.0001)$ (Table 5). The Youden index (0.536) and AUC (0.832) were largest when the inflammation grade was divided

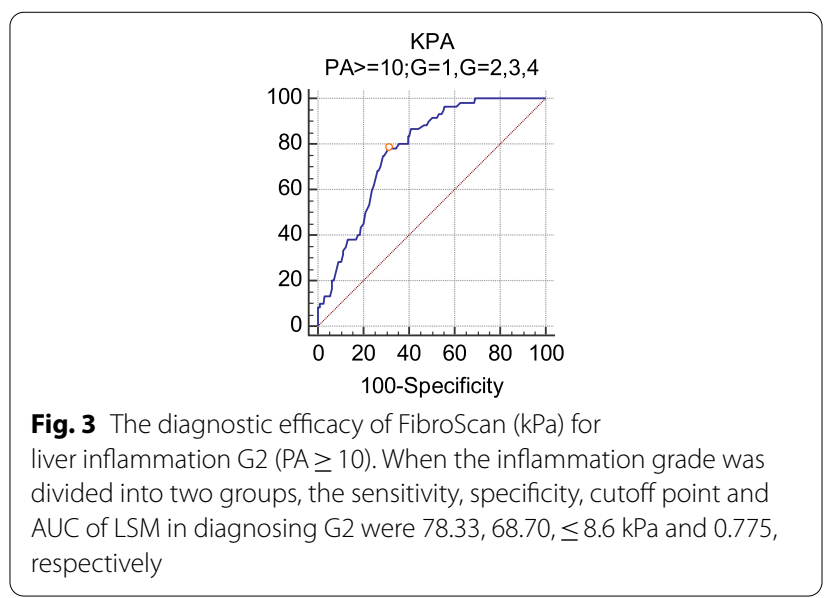

into two groups: $G=1,2,3$ and $G=4$. The sensitivity, specificity, cutoff point and AUC of LSM in diagnosing G4 were $87.43,66.21,>11 \mathrm{kPa}$ and 0.832 , respectively (Fig. 1). When the inflammation grade was redivided $(G=3,4$ and $G=1,2)$, the sensitivity, specificity, cutoff point and AUC of LSM in diagnosing G3 were 75.59, $75.51, \leq 9.8 \mathrm{kPa}$ and 0.818 , respectively (Fig. 2). When the inflammation grade was divided again $(\mathrm{G}=2,3,4$ and $\mathrm{G}=1$ ), the sensitivity, specificity, cutoff point and AUC of LSM in diagnosing G2 were $78.33,68.70, \leq 8.6 \mathrm{kPa}$ and 0.775 , respectively (Fig. 3).

There were significant differences in LSM among the liver fibrosis grades $(P<0.0001)$ (Table 4$)$. The Youden index (0.513) and AUC (0.826) were largest when the liver fibrosis staging was divided into $S=0,1,2$ and $S=3,4$. The sensitivity, specificity, cutoff point and AUC of LSM in diagnosing $\mathrm{S} 3$ were $77.45,73.88, \leq 10.4 \mathrm{kPa}$ and 0.826 , respectively. When the liver fibrosis stage was divided into two groups, $S=1,2,3,4$ and $S=0$, the sensitivity, specificity, cutoff point and AUC of LSM in diagnosing S1 were 83.33, 67.69, $\leq 8.6 \mathrm{kPa}$ and 0.791 , respectively. When the liver fibrosis stage was divided into $S=0,1,2$, 3 and $S=4$, the sensitivity, specificity, cutoff point and AUC of LSM in diagnosing S4 were 83.05, 65.37, > $11 \mathrm{kPa}$ 
and 0.807 , respectively. When the liver fibrosis stage was divided into $S=0,1$ and $S=2,3,4$, the sensitivity, specificity, cutoff point and the AUC of LSM in diagnosing S2 was $74.19,71.43, \leq 8.6 \mathrm{kPa}$ and 0.789 , respectively.

\section{Discussion}

Active inflammation promotes the development of fibrosis in CHB. Liver biopsy of chronic hepatitis showing variable necrotizing inflammation and/or fibrosis plays an important role in staging and grading CHB [20]. Despite its superiority of assessing both fibrosis and inflammation in CHB [8], liver biopsy is far from an ideal gold standard because of its invasiveness, risk of complications, patient discomfort and possible unavailability due to expertise requirements [21]. Staging CHB based on its severity using noninvasive tests such as elastography is important for guiding surveillance and assisting with treatment decisions [8]. Noninvasive tests are being increasingly incorporated into both national and international guidelines. With its good diagnostic accuracy for significant liver fibrosis and its excellent diagnostic accuracy for liver cirrhosis [22, 23], FibroScan has been widely applied. In addition to reflecting liver fibrosis, the LSM value by FibroScan (with liver biopsy as the reference standard) should also reflect changes in liver inflammation to some extent. Although it has been proven that necrotizing inflammation can lead to an increase in LSM in CHB [12, 24], more strong, persuasive clinical research evidence must be collected.

In our study, the main demographic and clinical characteristics of the 1185 patients with CHB were consistent with previous research reports, showing good representativeness $[1,7,25]$ : the male patients accounted for $75 \%$, the median age of $\mathrm{HBeAg}$-negative $\mathrm{CHB}$ patients was older than that of $\mathrm{HBeAg}$ - positive $\mathrm{CHB}$ patients $(P<0.001)$, the incidence of $\mathrm{CHB}$ complicated with hepatic steatosis $r$ in men was higher $(84 \%)$ than that in women $(16 \%)(P<0.001)$, the incidence of the degree of hepatic steatosis in $\mathrm{HBeAg}$ negative group was higher than that in $\mathrm{HBeAg}$ positive group $(P=0.004)$, the means \pm SD of ALT levels were higher in the HBeAgpositive patients than in the HBeAg-negative patients $(P=0.005)$, the patients with a normal level range of bilirubin accounted for $85 \%$ of the total while few patients had high bilirubin levels that affect liver stiffness, and the level of HBV DNA in the HBeAg-positive patients was higher than that in the HBeAg-negative patients $(P<0.001)$. More importantly, liver biopsy showed no difference in inflammation $(P=0.082)$ or fibrosis $(P=0.177)$ in patients with $\mathrm{CHB}$, regardless of whether they were complicated by hepatic steatosis, which further supported the view that the presence of steatosis in $\mathrm{CHB}$ patients does not lead to differences in the histopathological findings [26].

Usually, the performance of noninvasive diagnostic methods for liver diseases is evaluated by calculating the AUC using liver biopsy as the reference standard [11].

Accordingly, the quality of liver biopsy specimens is very important. It is recommended that if applicable, the presence of fewer than 11 PAs be noted in the pathology report, with recognition that the diagnosis, grading, and staging may be incorrect due to an insufficient sample size $[8,27]$. Good evidence shows that a biopsy containing 10 or fewer portal tracts results in underestimation of both the severity of the fibrosis stage and of the inflammatory grade in chronic viral hepatitis [28]. Therefore, for medical liver biopsies, the core of tissue should be intact and of sufficient size to demonstrate the lobular architecture of the liver over several portal tracts, which has been further emphasized in the recent guidelines of liver biopsy in clinical practice issued by the British Society of Gastroenterology, the Royal College of Radiologists and the Royal College of Pathology [29]. In our 1185 liver biopsy specimens of chronic hepatitis B, $82 \%$ had more than 10 PAs, and only $18 \%$ had fewer than 10 PAs, which provided a reliable guarantee for non-invasive diagnosis of liver inflammation or fibrosis by FibroScan.

A reliable TE assessment was defined as an assessment fulfilling three characteristics: a minimum of 10 readings, a success rate of measurements ("shots") $\geq 60 \%$ and an $\mathrm{IQR} /$ median ratio $(\mathrm{IQR} / \mathrm{M})$ of $\leq 0.30[16,22,30,31]$. The reliability of liver stiffness evaluations depend on the IQR/M according to the median liver stiffness level [17], so it is necessary to achieve a "very reliable" IQR/M $(\leq 0.10)$ or the "reliable" IQR/M (0.10-0.30) in the FibroScan test to the greatest extent possible. With a test success rate of over $90 \%$, the LSM reliability results of IQR/M in the 1185 patients with $\mathrm{CHB}$ were $70 \%(\mathrm{IQR} / \mathrm{M} \leq 0.10)$, $23 \%(0.10<\mathrm{IQR} / \mathrm{M} \leq 0.15), 4 \%(0.15<\mathrm{IQR} / \mathrm{M} \leq 0.20)$ and $3 \%(0.20<\mathrm{IQR} / \mathrm{M} \leq 0.3)$, respectively. The grade of liver inflammation was positively correlated with the stage of fibrosis (the Spearman's rho $=0.829, P<0.001$ ). Especially the liver inflammation of patients with cirrhosis (S4) is mostly G4, G1 and G2 are rare.

In CHB patients with liver fibrosis at stage $\mathrm{S} 2$ or above, the LSM values rised with the increase of the grades of liver inflammation. In S2 stage of liver fibrosis, there was significant difference in LSM values between different grades of liver inflammation $(\mathrm{F}=10.664, P<0.001)$. There was significant difference in LSM values between G1 inflammation and G3 inflammation $(P=0.01)$ and that between G2 inflammation and G3 inflammation $(P<0.001)$. In the S3 stage of liver fibrosis, there was significant difference in LSM values between different grades of liver inflammation $(\mathrm{F}=6.194, P=0.002)$. There 
was no statistical difference in LSM values between G2 inflammation and G3 inflammation $(P=0.051)$, which may be related to a small number of patients with G2 inflammation; there was significant difference in LSM values between G2 inflammation and G4 inflammation $(P=0.002)$ and that between G3 inflammation and G4 inflammation $(P=0.006)$. There was significant difference in LSM values between G3 inflammation and G4 inflammation in $\mathrm{S} 4$ stage of liver fibrosis $(P<0.001)$. It seemed to indicate that different grades of inflammation will have significant rise in LSM values within the same fibrosis stage.In order to further confirm this, we also carried out Spearman rank correlation coefficient analysis. It showed that LSM values were positively correlated with liver inflammation grade and fibrosis stage, and the Spearman's rho is 0.579 and 0.593 respectively $(P<0.001)$. Therefore, LSM value is not only related to the stage of liver fibrosis, but also related to the grade of liver inflammation. That was to say, LSM value could also reflect the grade of liver inflammation to a certain extent.

Then, the sensitivity, specificity, misdiagnosis rate, cutoff point and AUC of LSM were compared individually, and significant differences in the LSMs were noted among different grades of liver inflammation in the 1185 CHB patients $(P<0.0001)$. The cutoff points and AUCs of LSMs for the diagnosis of G2, G3, and G4 were $9.6 \mathrm{kPa}$ and $0.743,9.7 \mathrm{kPa}$ and 0.807 , respectively, and $11.4 \mathrm{kPa}$ and 0.838 , respectively; that is, FibroScan could diagnose G2, G3, and G4 liver inflammation in CHB patients with LSM values of $9.6 \mathrm{kPa}, 9.7 \mathrm{kPa}$ and $11.4 \mathrm{kPa}$, respectively.

Considering that the number of PAs in liver biopsy tissues will affect the pathological diagnosis of inflammation or fibrosis of liver tissues, we also analyzed the diagnostic efficacy of FibroScan (LSM) for liver inflammation or fibrosis when $P A \geq 10$ in the liver tissues of these patients. There were significant differences in the LSMs among different grades of liver inflammation $(P<0.0001)$. The cutoff points and the AUCs of the LSMs for the diagnosis of G2, G3, and G4 were $8.6 \mathrm{kPa}$ and $0.775,9.8 \mathrm{kPa}$ and 0.818 , and $11 \mathrm{kPa}$ and 0.832 , respectively. Significant differences were observed in the LSMs across the different stages of liver fibrosis $(P<0.0001)$. The cutoff points and the AUCs of the LSMs for the diagnosis of S2, S3, and S4 were $8.6 \mathrm{kPa}$ and $0.789,10.4 \mathrm{kPa}$ and 0.826 , and $11 \mathrm{kPa}$ and 0.807 , respectively; that is, FibroScan could diagnose G2, G3, and G4 liver inflammation in CHB patients with LSM values of $8.6 \mathrm{kPa}, 9.8 \mathrm{kPa}$ and $11.0 \mathrm{kPa}$, respectively. In addition, the efficacy of FibroScan for the noninvasive diagnosis of liver fibrosis, especially S4, was basically consistent with international reports or guideline recommendations [22, 32]. Most interestingly, the LSM cutoff point for G4 liver inflammation was $11.0 \mathrm{kPa}$, which was equal to that $(11.0 \mathrm{kPa})$ for the diagnosis of S4 liver fibrosis. Therefore, we believe that FibroScan has certain potential for the noninvasive diagnosis of $\mathrm{CHB}$, regardless of whether liver fibrosis or liver inflammation is being evaluated. Relevant studies had shown that LSM could diagnose different stages of liver fibrosis in patients with $\mathrm{CHB}$ after 78 weeks of antiviral treatment, and the decrease of LSM absolute value could reflect the remission of liver inflammation [33]. The latest study found that Liver inflammation activity over $2(\mathrm{OR}=3.53)$ was an independent risk factor for misdiagnosis of fibrosis stage using FibroScan, patients with liver inflammation activity $\geq 2$ showed higher LSM values using FibroScan and higher rates of misdiagnosis of fibrosis stage, whereas the diagnostic performance of FibroScan for different fibrosis stages was significantly lower than that in patients with inflammation activity $<2($ all $P<0.05)$ [34]. It is expert opinion that each patient becomes his or her own control, using the stiffness delta changes over time to evaluate the efficacy of the treatment or the progression of disease-remembering that the measurement reflects stiffness and not fibrosis [35]. Therefore, suppose of we see LSM 11 we consider that both liver inflammation and fibrosis exist. At this time, it is strongly recommended that these CHB patients should conduct liver biopsy to clearly distinguish the grade of liver inflammation and the stage of liver fibrosis, and establish the exact point of liver inflammation or fibrosis corresponding to LSM, so as to provide a real-time, dynamic and noninvasive reliable tracking means for long-term standardized treatment efficacy judgment or disease progress monitoring.

Treatment decisions for CHB sometimes depend on the presence of necroinflammation rather than fibrosis, so the challenge is now to decide on how best to apply validated noninvasive tests in $\mathrm{CHB}$ management [36]. ALT is used as a control liver test and serves as a nonspecific biomarker of liver injury, and serial testing of ALT levels is needed to guide treatment decisions for $\mathrm{CHB}$ patients [10]. Due to the discomfort of blood sample collection, the poor correlation with the degree of liver disease in CHB patients, and the fact that this measurement that may fail to identify patients with necroinflammatory activity or fibrosis [37, 38], serum ALT is still not the ideal biomarker for assessing the degree of liver injury in CHB patients.

Comparatively, owing to its noninvasive, rapid and dynamic nature, we should not overlook the superiority of FibroScan for the evaluation of liver inflammation in CHB patients. In some reports or guidelines on the noninvasive diagnosis of liver fibrosis by FibroScan, it has been suggested that the LSM cutoff value should be adapted to the ALT level since ALT levels tend to influence the LSM in CHB [39] and because ALT increases the LSM value in FibroScan and is an important factor 
or confounding factor affecting the accuracy of LSM, thus reducing its diagnostic efficiency [22, 40]. Since elevated ALT levels can reflect liver injury to some extent and necrotizing inflammation can lead to an increase in LSMs in CHB patients [24], why do we not deduce that the LSM value of FibroScan may reflect the degree of liver inflammation in addition to liver fibrosis? On the other hand, studies have shown that sustained HBV suppression with antiviral treatment can lead to a reduction in necroinflammatory activity and improvement in fibrosis stage, and CHB patients can have a significant reduction in liver stiffness during nuleos $(\mathrm{t})$ ide analog treatment, even when there is little or no improvement in fibrosis according to the histologic findings [41, 42]. Therefore, the impact of ALT normalization by antiviral therapy has to be considered in the interpretation of the noninvasive liver fibrosis assessment results [11], which indicates that the LSM value of FibroScan reflects the recovery of liver inflammation rather than liver fibrosis in $\mathrm{CHB}$ patients after antiviral therapy at a certain period of time. Remarkably, different ALT levels did not affect the accuracy of the LSM for the diagnosis of liver inflammation in our study $(P>0.05)$, so the influence of ALT on LSM should not be considered too heavily, and more attention should be given to the effect of liver inflammation on LSM. Regardless of whether liver inflammation or fibrosis is present, a decrease in the LSMs of CHB patients are welcome.

In summary, a reliance on abnormal liver function tests unfortunately causes most patients with significant liver injury to be missed [4], so noninvasive diagnostic techniques are needed to aid in $\mathrm{CHB}$ diagnosis and treatment monitoring. As the earliest and most extensively evaluated elastographic method for liver stiffness, FibroScan has certain potential for the noninvasive diagnosis of liver inflammation in CHB. The liver inflammation of $\mathrm{CHB}$ is accompanied by the occurrence and development of liver fibrosis, which was also proved in this study. It is difficult for LSM to exclude liver inflammation as an important participant in noninvasive diagnosis of liver fibrosis. In that case, we could expand the new use of LSMs for noninvasive diagnosis of liver inflammation, which was the goal of this study. This study showed that FibroScan might be a noninvasive diagnostic method for liver inflammation in $\mathrm{CHB}$ patients, which was better not only to expand the application field of the noninvasive diagnostic techniques of Fibroscan, but also to analyze the clinical connotation of LSM from different levels. For example, a rapid decrease of LSM in a short time after antiviral therapy is not likely to represent the remission or reversal of liver fibrosis, but more likely to be the improvement of liver inflammation in our view.

\section{Limitations}

There were still some defects in our study, especially how to adjust the impact of liver fibrosis on the readings were not clear, which is also the direction of further research in the future. On the other hand, this was a single-center retrospective study, so these findings need to be further verified by a multicenter prospective study.

\section{Conclusions}

In conclusion, the grade of liver inflammation was positively correlated with the stage of fibrosis, different grades of inflammation will have significant rise in LSM values within the same fibrosis stage. Based on the good quality of liver biopsy specimens ( $\mathrm{PA} \geq 10$ ), our single-center large sample data analysis showed that LSM cutoff points of $8.6 \mathrm{kPa}, 9.8 \mathrm{kPa}$ and $11.0 \mathrm{kPa}$ were effective in the diagnosis of G2, G3 and G4 liver inflammation in patients with $\mathrm{CHB}$, respectively. These results preliminarily showed that FibroScan could evaluate liver inflammation in CHB patients noninvasively, which is worthy of further clinical verification and improvement.

\section{Supplementary Information}

The online version contains supplementary material available at https://doi. org/10.1186/s12876-022-02108-0.

Additional file 1. LSM corresponding to the liver inflammation grade, fibrosis stage and serum ALT and AST levels (examples for illustration).

\section{Acknowledgements}

We thank the patients and our team for clinical case observation, FibroScan test, ultrasound-guided liver biopsy and liver histopathology.

\section{Authors' contributions}

K.J., L.Z. and J.L.: contributed equally to this work. K.J.: conceived and designed the study, collected and analyzed the large sample data and wrote the main manuscript text. L.Z.: carried out the observation of clinical cases, the data induction, clinical trial registration and some writing of the paper. J.L.: carried out the observation of clinical cases and the part of the clinical trial registration. H.H.: carried out the observation of clinical cases. Q.H.: carried out the Fibroscan test and quality control of the results. T.Q., X.M., J.R., W.G. and Y.T.: carried out the observation of clinical cases. H.C., Y.Z., X.C., Y.X., Y.L. and H.L.: carried out the classification and analysis of Fibroscan test data. Z.L.: carried out the liver biopsy guided by ultrasound. L.X. and R.M.: carried out the pathological diagnosis of liver tissue. Q.J. and K.H.: carried out the literature search and data extraction. All authors read and approved the final manuscript.

\section{Funding}

The work was sponsored by the Foshan High Level Medical Key Discipline Construction Project and "Peak Climbing Plan" for Foshan High Level Hospital Construction (Foshan Municipal Government Office [2019] No. 1).

\section{Availability of data and materials}

The datasets used and/or analyzed during the current study are available from the corresponding author on reasonable request. 


\section{Declarations}

\section{Ethics approval and consent to participate}

Approval was granted by the by the Ethics Committee of Foshan Hospital of Traditional Chinese Medicine (Ethics approval number:[2016]006).The study was conducted in accordance with the Declaration of Helsinki and International Good Clinical Practice Guidelines. All patients provided written notification consent and signed informed consent.

\section{Consent for publication}

Not applicable.

\section{Competing interests}

The authors declare that they have no competing interests.

\section{Author details}

'Department of Hepatology, Foshan Hospital of Traditional Chinese Medicine Affiliated to Guangzhou University of Chinese Medicine, No.6 Qinren Road, Chancheng District, Foshan 528000, Guangdong Province, China. ${ }^{2}$ Department of Ultrasound, Foshan Hospital of Traditional Chinese Medicine Affiliated to Guangzhou University of Chinese Medicine, No.6 Qinren Road, Chancheng District, Foshan 528000, Guangdong Province, China. ${ }^{3}$ Department of Pathology, Foshan Hospital of Traditional Chinese Medicine Affiliated to Guangzhou University of Chinese Medicine, No.6 Qinren Road, Chancheng District, Foshan 528000, Guangdong Province, China.

Received: 12 October 2021 Accepted: 20 January 2022

Published online: 29 January 2022

\section{References}

1. Schweitzer A, Horn J, Mikolajczyk RT, Krause G, Ott JJ. Estimations of worldwide prevalence of chronic hepatitis B virus infection: a systematic review of data published between 1965 and 2013. Lancet. 2015;386:1546-55.

2. Nayagam S, Thursz M, Sicuri E, et al. Requirements for global elimination of hepatitis B: a modelling study. Lancet Infect Dis. 2016;16:1399-408.

3. Tang LSY, Covert E, Wilson E, Kottilil S. Chronic hepatitis B infection: a review. JAMA. 2018;319:1802-13.

4. Harris R, Harman DJ, Card TR, Aithal GP, Guha IN. Prevalence of clinically significant liver disease within the general population, as defined by noninvasive markers of liver fibrosis: a systematic review. Lancet Gastroenterol Hepatol. 2017:2:288-97.

5. Ginès P, Graupera I, Lammert F, et al. Screening for liver fibrosis in the general population: a call for action. Lancet Gastroenterol Hepatol. 2016;1:256-60.

6. Shiha G, Ibrahim A, Helmy A, et al. Asian-Pacific Association for the Study of the Liver (APASL) consensus guidelines on invasive and non-invasive assessment of hepatic fibrosis: a 2016 update. Hepatol Int. 2017:11:1-30

7. European Association for the Study of the Liver. EASL 2017 Clinical practice guidelines on the management of hepatitis B virus infection. J Hepatol. 2017;67:3703-98

8. Terrault NA, Lok ASF, McMahon BJ, et al. Update on prevention, diagnosis, and treatment of chronic hepatitis B: AASLD 2018 hepatitis B guidance. Hepatology. 2018;67:1560-99.

9. Park JJ, Wong DK, Wahed AS, et al. Hepatitis B virus-specific and global T-cell dysfunction in chronic hepatitis B. Gastroenterology. 2016;150:684695.e5.

10. Li Y, Huang YS, Wang ZZ, et al. Systematic review with meta-analysis: the diagnostic accuracy of transient elastography for the staging of liver fibrosis in patients with chronic hepatitis B. Aliment Pharmacol Ther. 2016:43:458-69.

11. European Association for Study of Liver, Asociacion Latinoamericana para el Estudio del Higado. .EASL-ALEH clinical practice guidelines: non-invasive tests for evaluation of liver disease severity and prognosis. J Hepatol. 2015;63:237-64

12. Huang $R$, Jiang $N$, Yang $R$, et al. Fibroscan improves the diagnosis sensitivity of liver fibrosis in patients with chronic hepatitis B. Exp Ther Med. 2016;11:1673-7.
13. Castera L. Hepatitis B: are non-invasive markers of liver fibrosis reliable? Liver Int. 2014;34:91-6.

14. Hou J, Wang G, Wang F, et al. Guideline of prevention and treatment for chronic hepatitis B (2015 update). J Clin Transl Hepatol. 2017:5:297-318.

15. Jung KS, Kim SU, Ahn SH, et al. Risk assessment of hepatitis B virus-related hepatocellular carcinoma development using liver stiffness measurement (FibroScan). Hepatology. 2011;53:885-94.

16. Boursier J, Zarski JP, de Lédinghen $V$, et al. Determination of reliability criteria for liver stiffness evaluation by transient elastography. Hepatology. 2013;57:1182-91.

17. Bedossa P, Poynard T. An algorithm for the grading of activity in chronic hepatitis C. The METAVIR Cooperative Study Group. Hepatology. 1996:24:289-93.

18. Brunt EM. Grading and staging the histopathological lesions of chronic hepatitis: the Knodell histology activity index and beyond. Hepatology. 2000;31:241-6.

19. Kleiner DE, Brunt EM, Van Natta M, et al. Design and validation of a histological scoring system for nonalcoholic fatty liver disease. Hepatology. 2005:41:1313-21.

20. Mani $\mathrm{H}$, Kleiner DE. Liver biopsy findings in chronic hepatitis B. Hepatology. 2009;49:S61-71.

21. Bravo AA, Sheth SG, Chopra S. Liver biopsy. N Engl J Med. 2001;344:495-500.

22. Lim JK, Flamm SL, Singh S, Falck-Ytter YT. American Gastroenterological Association Institute guideline on the role of elastography in the evaluation of liver fibrosis. Gastroenterology. 2017;152:1536-43.

23. Herrmann $E$, de Lédinghen $V$, Cassinotto $C$, et al. Assessment of biopsyproven liver fibrosis by two-dimensional shear wave elastography: an individual patient data-based meta-analysis. Hepatology. 2018;67:260-72

24. Verveer C, Zondervan PE, ten Kate FJ, Hansen BE, Janssen HL, de Knegt RJ. Evaluation of transient elastography for fibrosis assessment compared with large biopsies in chronic hepatitis B and C. Liver Int. 2012;32:622-8.

25. Hadziyannis SJ. Natural history of chronic hepatitis B in Euro-Mediterranean and African countries. J Hepatol. 2011;55:183-91.

26. Yilmaz B, Koklu S, Buyukbayram H, et al. Chronic hepatitis B associated with hepatic steatosis, insulin resistance, necroinflammation and fibrosis. Afr Health Sci. 2015;15:714-8.

27. Rockey DC, Caldwell SH, Goodman ZD, Nelson RC, Smith AD. Liver biopsy. Hepatology. 2009;49:1017-44

28. Colloredo G, Guido M, Sonzogni A, et al. Impact of liver biopsy size on histological evaluation of chronic viral hepatitis: the smaller the sample, the milder the disease. J Hepatol. 2003:39:239-2344.

29. Neuberger J, Patel J, Caldwell H, et al. Guidelines on the use of liver biopsy in clinical practice from the British Society of Gastroenterology, the Royal College of Radiologists and the Royal College of Pathology(J). Gut. 2020;69:1382-403.

30. Kemp W, Levy M, Weltman M, Lubel J, Australian Liver Association (ALA) Australian Liver Association (ALA) expert consensus recommendations for the use of transient elastography in chronic viral hepatitis. J Gastroenterol Hepatol. 2015;30:453-62.

31. Boursier J, de Ledinghen V, Zarski JP, et al. Comparison of eight diagnostic algorithms for liver fibrosis in hepatitis C: new algorithms are more precise and entirely noninvasive. Hepatology. 2012;55:58-67.

32. Tapper EB, Lok AS. Use of liver imaging and biopsy in clinical practice. $N$ Engl J Med. 2017:377:756-68.

33. Dong $X Q, W u Z$, Li J, et al. Declining in liver stiffness cannot indicate fibrosis regression in patients with chronic hepatitis B: a 78-week prospective study. J Gastroenterol Hepatol. 2019;34:755-63.

34. Huang LL, Yu XP, Li JL, et al. Effect of liver inflammation on accuracy of FibroScan device in assessing liver fibrosis stage in patients with chronic hepatitis B virus infection. World J Gastroenterol. 2021;27:641-53.

35. Barr RG, Wilson SR, Rubens D, Garcia-Tsao G, Ferraioli G. Update to the Society of Radiologists in ultrasound liver elastography consensus statement. Radiology. 2020;296:263-74.

36. Wong GL, Wong VW, Choi PC, et al. On-treatment monitoring of liver fibrosis with transient elastography in chronic hepatitis B patients. Antivir Ther. 2011;16:165-72.

37. Keeffe EB, Dieterich DT, Han SHB, et al. A treatment algorithm for the management of chronic hepatitis B virus infection in the United States: 2008 update. Clin Gastroenterol Hepatol. 2008:6:1315-41. 
38. Pratt DS, Kaplan MM. Evaluation of abnormal liver-enzyme results in asymptomatic patients. N Engl J Med. 2000;342:1266-71.

39. Dietrich CF, Bamber J, Berzigotti A, et al. EFSUMB guidelines and recommendations on the clinical use of liver ultrasound elastography, update 2017 (short version). Ultraschall Med. 2017;38:377-94.

40. Ferraioli G, Wong VW, Castera L, et al. Liver ultrasound elastography: an update to the World Federation for Ultrasound in Medicine and Biology Guidelines and Recommendations. Ultrasound Med Biol. 2018;44:2419-40

41. Marcellin P, Gane E, Buti M, et al. Regression of cirrhosis during treatment with tenofovir disoproxil fumarate for chronic hepatitis B: a 5-year openlabel follow-up study. Lancet. 2013;381:468-75.

42. Liang $X$, Xie $Q$, Tan D, et al. Interpretation of liver stiffness measurementbased approach for the monitoring of hepatitis B patients with antiviral therapy: a 2-year prospective study. J Viral Hepat. 2018;25:296-305.

\section{Publisher's Note}

Springer Nature remains neutral with regard to jurisdictional claims in published maps and institutional affiliations.

- fast, convenient online submission

- thorough peer review by experienced researchers in your field

- rapid publication on acceptance

- support for research data, including large and complex data types

- gold Open Access which fosters wider collaboration and increased citations

- maximum visibility for your research: over 100M website views per year

At BMC, research is always in progress.

Learn more biomedcentral.com/submissions 